\title{
Clinical aspects of SARS-CoV-2 infection and vitamin D
}

\section{COVID-19 and the endocrine system: special issue for reviews in endocrine and metabolic disorders (Felipe Casaneuva, Editor in Chief) A. Giustina and JP Bilezikian, Guest Editors}

\author{
Leonardo Bandeira $^{1,2}$ (D) Marise Lazaretti-Castro ${ }^{1}$ (D) Neil Binkley $^{3}$ (D) \\ Accepted: 21 August 2021 / Published online: 24 September 2021 \\ (c) The Author(s), under exclusive licence to Springer Science+Business Media, LLC, part of Springer Nature 2021
}

\begin{abstract}
In December 2019, the first cases of severe acute respiratory syndrome due to a new coronavirus (SARS-Cov-2), later designated as Covid-19, were described in China. With rapid advance of the infection to several continents, in March 2020, WHO declared this to be a pandemic. In April 2020, the first papers suggesting a possible role of Vitamin D deficiency in the severity of this infection began to appear and dozens of articles evaluating a potential relationship of vitamin $\mathrm{D}$ with COVID have emerged subsequntly. This possibility was raised based on pre-existing evidence of the effects of Vitamin D on the immune system, and more specifically on acute respiratory viral infections. In addition, most Covid-19 victims belong to groups at risk for vitamin D deficiency such as the elderly, obese, chronically ill, and specific ethnic groups. Although with some contradictory reports exist, most observational and cohort studies find a relationship of low vitamin D status with greater Covid severity, others, including the few interventional studies available show inconsistent results. This paper aims to present the rapidly expanding literature to date regarding the clinical relevance of vitamin D in Covid-19 and, consequently, the reasonableness of avoiding its deficiency to keep the immune system able to respond in the best way to this acute viral infection. In the meantime, we wait for publication of several prospective randomized controlled studies that are underway, evaluating the effects of treatment with vitamin D or metabolites on the severity of Covid-19 outcomes.
\end{abstract}

Keywords Vitamin D · COVID-19 · SARS-CoV-2 $\cdot$ Respiratory infectious disease $\cdot$ Calcifediol $\cdot$ Calcitriol

\section{Introduction}

Active vitamin D (calcitriol) acts on the immune system, through its nuclear receptors on monocytes/macrophages (activating the synthesis of cathelicidin and defensins, which have an anti-microbial function) and on $\mathrm{T}$ and $\mathrm{B}$ lymphocytes (leading to greater production

Marise Lazaretti-Castro

marise.lazaretti@imabrasil.com.br

Leonardo Bandeira

leonardo.farias@grupofleury.com.br

Neil Binkley

nbinkley@wisc.edu

Universidade Federal de Sao Paulo, Sao Paulo, Brazil

2 Grupo Fleury, Recife, Brazil

3 School of Medicine and Public Health, University of Wisconsin, Madison, WI, USA of immunoglobulins, a reduction in pro-inflammatory cytokines, and an increase in anti-inflammatory cytokines) [1-6]. Some clinical data suggest there is possible evidence for protective vitamin $\mathrm{D}$ effects on pulmonary function and respiratory tract infections, like influenza and tuberculosis [7-13].

Vitamin D also inhibits expression of the renin-angiotensinaldosterone system, which is dysregulated in favor of angiotensin2 in SARS-CoV-2 infection, potentially contributing to multiple deleterious effects such as interstitial fibrosis, myocardial hypertrophy, endothelial fibrosis, increased inflammation, and vasoconstriction [14-19]. Lansiaux et al suggest COVID-19 mortality is negatively correlated with sunlight exposure [20], and therefore potentially related to vitamin D status. Indeed, the mortality rate due to COVID-19 appears to be higher in North American states, that have less sunlight exposure during the year, compared to the southern states [21]. Countries in Europe that have higher levels of vitamin D deficiency also have higher rates of infection and death by COVID-19 [22]. Thus, for 
all these reasons, it has been hypothesized that vitamin D status may have an association with COVID-19.

\section{Cross-sectional and cohort studies}

Multiple studies relating vitamin D status with COVID19 and outcomes have been published; a few examples are noted. A cross-sectional study evaluated the prevalence of hypovitaminosis $\mathrm{D}$ in 176 patients > age 60 (mean 72 years) with severe COVID-19 admitted to the Intensive Care Unit (ICU) in need of invasive ventilation due to Acute Respiratory Distress Syndrome (ARDS). The prevalence of hypovitaminosis $\mathrm{D}$ was very high in this population $(94 \%, 66 \%$ and $21 \%$, for 25 hydroxyvitamin D (25OHD) values $<30$, $<20$ and $<10 \mathrm{ng} / \mathrm{ml}$, respectively) [23].

An observational cohort from a tertiary academic hospital in Singapore evaluated the effect of the combination vitamin $\mathrm{D}$, magnesium, and vitamin B12 in patients over 50 years hospitalized for COVID-19. As the pandemic evolved, the institution's COVID-19 therapeutic protocol added a scheme with a single daily oral dose of $1.000 \mathrm{IU}$ of vitamin D3, $150 \mathrm{mg}$ of magnesium oxide, and $500 \mathrm{mg}$ vitamin B12 for up to 14 days in patients who initially did not indicate ICU. Data were collected from electronic health records. A group of 17 patients who received the regimen was compared to a control group of 26 patients. The group that received the scheme had less need for oxygen therapy and admission to the ICU [24].

Ling SF et al evaluated the relationship between vitamin D status and mortality in more than 900 adult patients hospitalized with COVID-19 in three UK hospitals. The mean age of the patients was greater than 70 years and the average level of $25 \mathrm{OHD}$ was less than $20 \mathrm{ng} / \mathrm{ml}$, thus characterizing most of them as vitamin $\mathrm{D}$ deficient. The results were divided into two cohorts (the first one including 444 participants with an average 25OHD level of $12 \mathrm{ng} / \mathrm{ml}$ recruited from one hospital; the second including 542 participants with an average 25OHD level of $17 \mathrm{ng} / \mathrm{ml}$ recruited from two other medical centers). Those who received high doses of cholecalciferol (at least $40.000 \mathrm{IU} /$ week) during hospitalization had a lower risk of death from the disease (first cohort OR 0.13, 95\% CI 0.05-0.35, p <0.001; second cohort OR $0.38,95 \%$ CI $0.17-0.84, p=0.018$ ) [25].

Other studies have not confirmed the association between vitamin D status and COVID-19. Hastie CE et al, using data from the UK biobank, assessed 449 COVID patients and found no relationship between the SARS-CoV-2 infection and 25OHD levels after adjustments for confounders $(\mathrm{OR}=$ $1.00,95 \% \mathrm{CI}=0.998-1.01, p=0.2$ ). However, an important limitation of this study is that serum 25OHD was obtained more than a decade before infection by COVID-19 (between 2006 and 2010). In this cross-sectional study, ethnicity was a risk factor for COVID-19 infection, with blacks and South Asians having more risk than whites $(p<0.001)$ [26]. A prospective cohort study, which also used data from the UK biobank, with a larger number of patients $(n=1,326)$ also found no relationship between 25OHD levels and the SARSCoV-2. Similarly, people from minority ethnicities, such as black and Asians, had a greater risk of presenting with COVID-19 [27]. These data suggest that the difference in the SARS-Cov-2 infection incidence among ethnicities is not explained by the 25OHD levels, and thus other factors must be involved such as genetic susceptibility and economic, social and behavioral differences [26, 27].

Brandao et al evaluated 13,930 participants who underwent COVID-19 RT-PCR (reverse transcriptase polymerase chain reaction) testing and compared the serum 25OHD levels between the groups with positive $(n=2,345)$ and negative $(n=11,585)$ results [28]. These study participants were men and women, between 18 and 90 years old from the city of Sao Paulo - Brazil, and the groups were matched for gender and age. The data were obtained from the database of a private clinical analysis laboratory that provides supplemental health services, so the population was mainly from a high socioeconomic level. Data collection took place over 6 months starting at the end of summer in the southern hemisphere and this could explain the high mean 25OHD levels in both groups $(>28 \mathrm{ng} / \mathrm{mL})$. A previous study held in the same city has previously demonstrated that the months following summer is a period when the population has a higher 25OHD level compared to winter/spring [29]. Besides, there was no information available regarding Vitamin D supplementation, and it is possible that many of them were taking it. Nevertheless, no differences on 25OHD levels were found between those with and without confirmed SARS-Cov-2 infection by RT-PCR (positive $28.8 \mathrm{ng} / \mathrm{ml}$ vs. negative 29.6 $\mathrm{ng} / \mathrm{ml}$, respectively; $p=0.08$ ) [28].

\section{Systematic reviews and metanalysis of observational studies}

Despite the discrepancy in observational results, meta-analyses support the hypothesis that an adequate vitamin $\mathrm{D}$ status positively influences COVID-19 outcomes. Liu $\mathrm{N}$ et al included 10 case-control studies, numbering more than 350 thousand individuals, most of them over 50 years old, in a meta-analysis. Despite high heterogeneity among the studies, overall vitamin D deficiency or insufficiency $(25 \mathrm{OHD}<30 \mathrm{ng} / \mathrm{ml}$ ) was associated with an increased risk of SARS-CoV-2 infection (OR = $1.43,95 \%$ CI $1.00-2.05$ ). Additionally, patients with confirmed COVID-19 had lower 25OHD levels than participants who did not have the disease. The difference between the groups was subtle but statistically significant (standard mean difference $\mathrm{SMD}=-0.37,95 \%$ CI -0.52 to -0.21$)$ [30]. 
An additional systematic review evaluated 9 studies, comprising 1.005 .042 participants. It is important to mention that one of these studies had a sample of 1 million participants while the other 8 were smaller studies with a maximum of 1.326 people included. Among the studies, 7 demonstrated a correlation between vitamin $\mathrm{D}$ status and COVID-19. Lower 25OHD levels were associated with a greater chance of COVID-19 infection, more severe presentation, more hospitalization and ICU admission, higher C-Reactive Protein (CRP) levels, and higher mortality. COVID-19 patients received less vitamin D supplementation than those who did not have the disease. Also, some factors were associated with worst outcomes such as age, male sex, high BMI, and presence of comorbidities [31].

A very recent metanalysis assessed 376 COVID-19 patients, with a mean age of 63.8 years and a very low mean $25 \mathrm{OHD}$ level of $8.7 \mathrm{ng} / \mathrm{ml}$, in 6 studies (2 Asian, 3 European, and 1 American). The lowest levels of 25OHD were found in Asian patients (average $=6.7 \mathrm{ng} / \mathrm{ml}$ ). Those who evolved with ARDS and/or mechanical ventilation requirement had lower levels of 25OHD (SMD -0.58, 95\% CI - 0.83 to -0.34, $p<0.001)$ compared to those with milder presentation [32]. Similarly to Liu et al systematic review [30], the difference between the groups was subtle but statistically different.

In another systematic review, Pereira $M$ et al included 27 studies in their analysis. More than 8.000 people with an average age of 58 participated. Vitamin D deficiency was not associated with a higher risk of infection by COVID-19, but those who had more severe presentation had a higher risk of vitamin D deficiency $(\mathrm{OR}=1.64,95 \% \mathrm{CI}=1.30-2.09)$. Vitamin D insufficiency was associated with more hospitalization and higher mortality due to COVID-19 [33].

In these studies relating circulating $25 \mathrm{OHD}$ concentration with COVID-19 outcomes, it is important to recognize the possibility that $25 \mathrm{OHD}$ may be a negative acute phase reactant with inflammation reducing the measured value. Clearly, acute inflammation from endotoxin administration [34] and surgical intervention [35] promptly reduce circulating 25OHD. Multiple other studies find a relationship of $25 \mathrm{OHD}$ with inflammation [36-38]. As such, it is possible that circulating 25OHD is a negative acute phase reactant with lower levels indicating greater inflammation. Given this, it is plausible that low 25OHD levels observed in COVID-19 patients reflect reverse causality, i.e., the SARS-CoV-2 infection causes low 25OHD. If this is indeed correct, lower 25OHD might be a marker of more severe disease. As such, while low 25OHD levels may indicate tissue or cellular level vitamin $\mathrm{D}$ deficiency that preceded infection and be causally related to adverse COVID-19 outcomes, it is also plausible that this is an association identifying individuals with more severe disease in whom poorer outcomes could be expected. Prospective randomized trials of vitamin D supplementation are needed to clarify this issue.

\section{Randomized controlled trials}

In a pilot randomized open-label trial performed in one Spanish center, Entrenas Castillo et al studied the effects of vitamin D treatment on patients with COVID19.. Seventy-six patients hospitalized with the infection (confirmed by chest X-ray and positive SARS-CoV-2 RTPCR test) were randomized into 2 groups. The first, with 50 patients, received high dose of calcifediol $(25-\mathrm{OH}-$ vitamin D3) orally on admission, on days 3 and 7, and then weekly. The 25 remaining patients were allocated to the control group. All patients also received an association of hydroxychloroquine and azithromycin, which at the beginning of the pandemic was used as a therapeutic regimen in some countries $[39,40]$. As a result, the group that took calcifediol had less need for ICU admission compared to the control group, with a notable statistical difference between them (OR $0.03,95 \%$ CI $0.003-0.25$, $p<0.001$ ) [41]. Although these results were very positive, there are some limitations to be considered, such as the small size of the sample and the lack of 25OHD concentrations at baseline and after treatment. Based on these good results, a multicenter randomized controlled trial using calcifediol is being carried out in 15 centers from Spain (see at Clinicaltrials.gov).

The largest published double-blind randomized control trial of vitamin $\mathrm{D}$ administration among hospitalized COVID-19 patients to date compared the effects of a single high dose of vitamin D vs placebo in 237 patients with criteria for hospitalization due to COVID-19 (respiratory rate greater than 24/minute, O2 saturation less than $93 \%$ while breathing room air, or risk factors for complications like heart disease, diabetes, systemic arterial hypertension, neoplasms, immunosuppression, pulmonary tuberculosis, obesity) in Brazil. Participants had a mean age of 56 years and serum $250 \mathrm{OHD}$ of $20.9 \mathrm{ng} / \mathrm{ml}$ at baseline. The intervention group took a single dose of 200,000 UI of cholecalciferol orally, which led to higher 25OHD levels compared to placebo before discharge (44.4 vs $19.8 \mathrm{ng} / \mathrm{ml}$, $p<0.001)$. Nevertheless, there was no difference between groups regarding the length of stay, mortality, admission to ICU, or need for mechanical ventilation [42]. Although the results of this trial did not show the benefits of vitamin D treatment in patients hospitalized with COVID-19 [43], it should be considered that the majority of the participants had vitamin D levels greater than $20 \mathrm{ng} / \mathrm{ml}$, which are specifically those who would have less benefit from replacement. In addition, critical patients were excluded from the analysis. This is important because other therapies tested on hospitalized patients with COVID-19, as dexamethasone and tocilizumab, have shown more benefits exactly in patients with more severe disease [44, 45]. 
Additionally, it is important to consider that vitamin D supplementation with physiologic doses to achieve widely accepted 25OHD levels considered adequate may not be the same as large pharmacologic doses. In this regard, it is plausible that high-dose vitamin D might have effects as a "drug" that are not observed with "supplementation" doses. Thus, it seems prudent to consider vitamin D supplementation studies as distinct from vitamin D pharmacologic dose treatment studies. Finally, it is also plausible that huge vitamin D doses might not have a potential pharmacologic effect when administered later in the disease course often in combination with extremely potent anti-inflammatory medications (i.e., dexamethasone). It may not be possible to further modulate inflammation in the setting of potent glucocorticoid therapy. Confounders such as this suggest that well designed animal model studies may well be essential to define the role(s) of vitamin D in COVID-19 infection.

The association between COVID-19 and vitamin D is a hot topic nowadays. There are currently more than 30 intervention studies underway or to be initiated on this subject registered at the US National Institute of Health (NIH) [46].

\section{Conclusion}

In summary, despite controversial data, vitamin D intake and adequate 25OHD status may be associated with a lower risk of COVID-19 and a better clinical outcome in infected patients. As there are no curative drugs currently for the treatment of the disease, vitamin $\mathrm{D}$ is an inexpensive and safe option that can be used as an adjuvant in the adopted therapeutic plan. Based on current evidence, it is not possible to establish an ideal serum 25OHD level and an amount of daily intake that could potentially help with the prevention and treatment of COVID-19. Randomized control trial data are scarce, with different forms of intervention (cholecalciferol and calcifediol) and divergent conclusions. However, considering the results from the meta-analysis that showed a lower risk of COVID-19 in people with $25 \mathrm{OHD}$ levels $>30 \mathrm{ng} /$ $\mathrm{ml}$ and the recommendations adopted by the various guidelines for the prevention and treatment of bone metabolic disease, it is reasonable to avoid vitamin D deficiency/insufficiency. Maintaining an adequate 25OHD status may be a good approach to help to prevent COVID-19 and to lower the risk of severe outcome in infected patients. Further research on the subject is needed.

Funding No funding was received to assist with the preparation of this manuscript.

\section{Declarations}

Conflicts of interest MLC is principal investigator in research protocol on the epidemiology of vitamin D deficiency in Brazil sponsored by Mantecorp. NB and LB: No conflicts of interest

\section{References}

1. Baeke F, Takiishi T, Korf H, et al. Vitamin D: modulator of the immune system. Curr Opin Pharmacol. 2010;10(4):482-96.

2. Chun RF, Liu PT, Modlin RL, et al. Impact of vitamin D on immune function: lessons learned from genome-wide analysis. Front Physiol. 2014;5:151.

3. Cantorna MT, Snyder L, Lin YD, et al. Vitamin D and 1,25(OH)2D regulation of T cells. Nutrients. 2015;7(4):3011-21.

4. Santos RND, Maeda SS, Jardim JR, et al. Reasons to avoid vitamin D deficiency during COVID-19 pandemic. Arch Endocrinol Metab. 2020.

5. Bouillon R, Marcocci C, Carmeliet G, et al. Skeletal and extraskeletal actions of Vitamin D: current evidence and outstanding questions. Endocr Rev. 2019;40(4):1109-51.

6. Liu PT, Stenger S, Li H, et al. Toll-like receptor triggering of a vitamin D-mediated human antimicrobial response. Science. 2006;311(5768):1770-3.

7. Laaksi I, Ruohola JP, Tuohimaa P, et al. An association of serum vitamin $\mathrm{D}$ concentrations $<40 \mathrm{nmol} / \mathrm{L}$ with acute respiratory tract infection in young finnish men. Am J Clin Nutr. 2007;86(3):714-7.

8. Martineau AR, Jolliffe DA, Hooper RL, et al. Vitamin D supplementation to prevent acute respiratory tract infections: systematic review and meta-analysis of individual participant data. Bmj. 2017;356:i6583.

9. Urashima M, Segawa T, Okazaki M, et al. Randomized trial of vitamin D supplementation to prevent seasonal influenza $\mathrm{A}$ in schoolchildren. Am J Clin Nutr. 2010;91(5):1255-60.

10. Aibana O, Huang CC, Aboud S, et al. Vitamin D status and risk of incident tuberculosis disease: A nested case-control study, systematic review, and individual-participant data meta-analysis. PLoS Med. 2019;16(9):e1002907.

11. Tenforde MW, Yadav A, Dowdy DW, et al. Vitamin A and D deficiencies associated with incident tuberculosis in HIV-infected patients initiating antiretroviral therapy in multinational casecohort study. J Acquir Immune Defic Syndr. 2017;75(3):e71-9.

12. Wu HX, Xiong XF, Zhu M, et al. Effects of vitamin D supplementation on the outcomes of patients with pulmonary tuberculosis: a systematic review and meta-analysis. BMC Pulm Med. 2018;18(1): 108 .

13. Nolasco R, Moreira LD, Bocalini DS, et al. Effects of vitamin D supplementation on pulmonary function in postmenopausal women following an aquatic exercise program. Arch Endocrinol Metab. 2017;61(1):28-35.

14. Giménez VMM, Sanz RL, Marón FJM, et al. Vitamin D-RAAS connection: An integrative standpoint into cardiovascular and neuroinflammatory disorders. Curr Protein Pept Sci. 2020;21(10):948-54.

15. Simões e Silva AC, Silveira KD, Ferreira AJ, et al. ACE2, angiotensin-(1-7) and $\mathrm{M}$ as receptor axis in inflammation and fibrosis. Br J Pharmacol. 2013;169(3):477-92.

16. Bourgonje AR, Abdulle AE, Timens W, et al. Angiotensinconverting enzyme 2 (ACE2), SARS-CoV-2 and the pathophysiology of coronavirus disease 2019 (COVID-19). J Pathol. 2020;251(3):228-48.

17. Reynolds HR, Adhikari S, Pulgarin C, et al. Renin-AngiotensinAldosterone System Inhibitors and Risk of Covid-19. N Engl J Med. 2020;382(25):2441-8.

18. Verdecchia P, Cavallini C, Spanevello A, et al. The pivotal link between ACE2 deficiency and SARS-CoV-2 infection. Eur J Intern Med. 2020;76:14-20.

19. Bilezikian JP, Bikle D, Hewison M, et al. Mechanisms in endocrinology: Vitamin D and COVID-19. Eur J Endocrinol. 2020;183(5):R133-r47. 
20. Lansiaux É, Pébaÿ PP, Picard JL, et al. Covid-19 and vit-d: Disease mortality negatively correlates with sunlight exposure. Spat Spatiotemporal Epidemiol. 2020;35:100362.

21. Marik PE, Kory P, Varon J. Does vitamin D status impact mortality from SARS-CoV-2 infection? Med Drug Discov. 2020;6:100041.

22. Lanham-New SA, Webb AR, Cashman KD, et al. Vitamin D and SARS-CoV-2 virus/COVID-19 disease. BMJ Nutrition, Prevention \& Health. 2020;3(1):106-10.

23. Gonçalves TJM, Gonçalves S, Guarnieri A, et al. Prevalence of obesity and hypovitaminosis D in elderly with severe acute respiratory syndrome coronavirus 2 (SARS-CoV-2). Clin Nutr ESPEN. 2020;40:110-4.

24. Tan CW, Ho LP, Kalimuddin S, et al. Cohort study to evaluate the effect of vitamin $\mathrm{D}$, magnesium, and vitamin $\mathrm{B}(12)$ in combination on progression to severe outcomes in older patients with coronavirus (COVID-19). Nutrition. 2020;79-80:111017.

25. Ling SF, Broad E, Murphy R, et al. High-dose cholecalciferol booster therapy is associated with a reduced risk of mortality in patients with COVID-19: A cross-sectional multi-centre observational study. Nutrients. 2020;12(12).

26. Hastie CE, Mackay DF, Ho F, et al. Vitamin D concentrations and COVID-19 infection in UK Biobank. Diabetes Metab Syndr. 2020;14(4):561-5.

27. Raisi-Estabragh Z, McCracken C, Bethell MS, et al. Greater risk of severe COVID-19 in Black, Asian and Minority Ethnic populations is not explained by cardiometabolic, socioeconomic or behavioural factors, or by $25(\mathrm{OH})$-vitamin D status: study of 1326 cases from the UK Biobank. J Public Health (Oxf). 2020;42(3):451-60.

28. Brandão C, Chiamolera MI, Biscolla RPM, et al. No association between vitamin D status and COVID-19 infection in São Paulo, Brazil. Arch Endocrinol Metab. 2021.

29. Maeda SS, Saraiva GL, Kunii IS, et al. Factors affecting vitamin D status in different populations in the city of São Paulo, Brazil: the São PAulo vitamin D Evaluation Study (SPADES). BMC Endocr Disord. 2013;13:14.

30. Liu N, Sun J, Wang X, et al. Low vitamin D status is associated with coronavirus disease 2019 outcomes: a systematic review and meta-analysis. Int J Infect Dis. 2021;104:58-64.

31. Yisak H, Ewunetei A, Kefale B, et al. Effects of Vitamin D on COVID-19 Infection and Prognosis: A Systematic Review. Risk Manag Healthc Policy. 2021;14:31-8.

32. Munshi R, Hussein MH, Toraih EA, et al. Vitamin D insufficiency as a potential culprit in critical COVID-19 patients. J Med Virol. 2021;93(2):733-40.

33. Pereira M, Dantas Damascena A, Galvão Azevedo LM, et al. Vitamin D deficiency aggravates COVID-19: systematic review and meta-analysis. Crit Rev Food Sci Nutr. 2020:1-9.
34. Smolders J, van den Ouweland J, Geven C, et al. Letter to the Editor: Vitamin D deficiency in COVID-19: Mixing up cause and consequence. Metabolism. 2021;115:154434.

35. Binkley N, Coursin D, Krueger D, et al. Surgery alters parameters of vitamin D status and other laboratory results. Osteoporos Int. 2017;28(3):1013-20.

36. Ghashut RA, Talwar D, Kinsella J, et al. The effect of the systemic inflammatory response on plasma vitamin $25(\mathrm{OH}) \mathrm{D}$ concentrations adjusted for albumin. PLoS One. 2014;9(3):e92614.

37. Reid D, Toole BJ, Knox S, et al. The relation between acute changes in the systemic inflammatory response and plasma 25-hydroxyvitamin D concentrations after elective knee arthroplasty. Am J Clin Nutr. 2011;93(5):1006-11.

38. Thurnham DI. Plasma 25-Hydroxy-Cholecalciferol (Vitamin D) is Depressed by Inflammation: Implications and Parallels with Other Micronutrients. Sight and Life. 2011:38-50.

39. https://www.aemps.gob.es/la-aemps/ultima-informacionde-la-aemps-acerca-del-covid\%E2\% 80\% 9119/tratamientosdisponibles-para-el-manejo-de-la-infeccion-respiratoria-por-sarscov-2/?lang=en. Agencia Española de Medicamentos y Productos Sanitarios. 2020.

40. Yao X, Ye F, Zhang M, et al. In vitro antiviral activity and projection of optimized dosing design of hydroxychloroquine for the treatment of severe acute respiratory syndrome coronavirus 2 (SARS-CoV-2). Clin Infect Dis. 2020;71(15):732-9.

41. Castillo ME, Costa LME, Barrios JMV, et al. Effect of calcifediol treatment and best available therapy versus best available therapy on intensive care unit admission and mortality among patients hospitalized for COVID-19: A pilot randomized clinical study. J Steroid Biochem Mol Biol. 2020;203:105751.

42. Murai IH, Fernandes AL, Sales LP, et al. Effect of a single high dose of vitamin D3 on hospital length of stay in patients with moderate to severe COVID-19: A randomized clinical trial. Jama. 2021.

43. Leaf DE, Ginde AA. Vitamin D3 to Treat COVID-19: different disease, same answer. Jama. 2021;325(11):1047-8.

44. Horby P, Lim WS, Emberson JR, et al. Dexamethasone in Hospitalized Patients with Covid-19 - Preliminary Report. N Engl J Med. 2020.

45. Salama C, Han J, Yau L, et al. Tocilizumab in Patients Hospitalized with Covid-19 Pneumonia. N Engl J Med. 2021;384(1):20-30.

46. https://clinicaltrials.gov/ct $2 /$ results?term $=V i t a m i n+D \&$ cond $=$ COVID $-19 \&$ recrs $=b \&$ recrs $=a \&$ recrs $=f \&$ recrs $=d \&$ age $\_v=\&$ gndr $=\&$ type $=$ Intr\&rslt $=\&$ Search $=$ Apply

Publisher's Note Springer Nature remains neutral with regard to jurisdictional claims in published maps and institutional affiliations. 